\title{
Model the Uncertainty in Target Recognition using Possiblized Bayes' Theorem
}

\author{
Wei Mei, Ganlin Shan, Chunping Wang \\ Dept. of Electronic Engr., \\ Shijiazhuang Mech. Engr. College \\ Shijiazhuang, P.R. China \\ meiwei@sina.com
}

\begin{abstract}
The major source of uncertainty in target recognition consists of two parts. One is about feature extraction from observation data and another is about the rule definition between target type and feature. While the former can be naturally captured in the form of statistics, it is our opinion that the latter should be defined by using possibility since exact probability assignment is in general impossible. This paper addresses target recognition within the Bayesian framework while reinterpreting the likelihood of Bayes' theorem as a possibility. It leads to an open structure of feature database, which can exempt the reconstruction of feature database of the Bayesian classifier when new feature rules need to be included. An example of target recognition using attribute data from an electronic support measure (ESM) shows that the proposed method has competitive performance with the conventional Bayesian classifier.
\end{abstract}

Keywords-Possibility theory; Fuzzy inference systems; Target recognition; Bayesian, Conditional probability; Likelihood

\section{INTRODUCTION}

Target tracking and recognition are two critical tasks of battlefield surveillance systems. The latter is usually considered to be more challenging for the more sophisticated uncertainty involved. Up to now, a variety of approaches, such as the Bayesian method [1, 2] the Dempster-Shafer method [2, 3], fuzzy set theories [4], and the neural network method [5] among others, have been developed to solve this problem. Among them, the Bayesian method remains the most popular, as it offers the most efficient and theoretically justifiable method of managing uncertainty in situations similar to target recognition.

This paper addresses the problem of target recognition with focus on uncertainty modeling. It is natural to describe the uncertainty of recognizing a target by its posterior type probability, from which a hard decision can finally be made. However, probability is by far the only uncertainty that need to be handled in the process of target recognition. In fact, there are two stages in target recognition with different kinds of uncertainty involved. The first stage is to define the function between the observed data and the feature selected for recognizing a certain target while the second step is about the relation of the target feature and the target type. It is very often that the uncertainty involved in the first stage can be given in

This work was supported in part by the Chinese NSFC under Grant 61141009 . the form of statistics, but in the second stage it can hardly be given in statistics. Instead it should be regarded as a possibility. The fusion of the two different kinds of uncertain information can be achieved by using the Bayesian updating procedure but with the likelihood function interpreted in terms of possibility as in [6-8].

After a discussion of the proposed method of uncertainty modeling for target recognition in Section 2, we show in Section 3 by a simulated example that the proposed classifier succeeded in recognizing a noncooperative target using attribute data from an electronic support measure (ESM). Section 4 concludes the paper.

\section{UNCERTAINTY MODELING FOR TARGET RECOGNITION}

The major source of uncertainty in target recognition arises from feature mapping, which consists of two stages as illustrated in Fig. 1 [9]. The first stage, uncertain mapping I, is to select/extract features from observation information. The second stage, uncertain mapping II, is to define the uncertain mapping between the feature space and the type space.

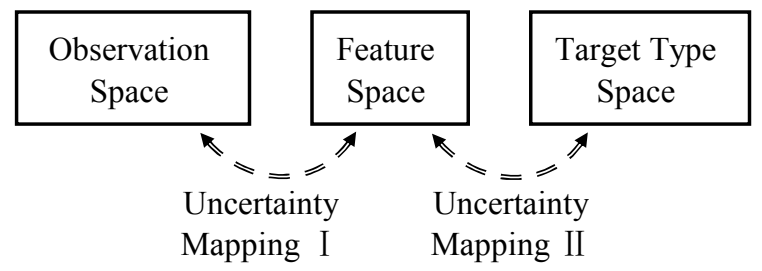

Figure 1. Two stages of uncertainty mapping in target recognition

From the conventional Bayesian viewpoint, the posterior type probability of a target being recognized could be updated using Bayes' rule of conditioning as follows:

$$
p\left(c_{i} \mid z_{1: k}\right)=\alpha p\left(c_{i} \mid z_{1: k-1}\right) p\left(z_{k} \mid c_{i}, z_{1: k-1}\right),
$$

where $c_{i}$ denotes a type $i$ target that belongs to the recognition frame $C=\left\{c_{i} \mid i=1, \ldots, n\right\} \cdot \boldsymbol{z}_{k}$ is the feature observation at time $k$, provided by a attribute sensor, e.g. an ESM. $\boldsymbol{z}_{1: k}=\left\{\boldsymbol{z}_{1}, \boldsymbol{z}_{2}, \cdots, \boldsymbol{z}_{k}\right\}$ is the cumulative set of the observations 
up to time $k$, and $\alpha$ is a normalization constant such that the values of $p\left(c_{i} \mid \boldsymbol{z}_{1: k}\right)$ sum to 1 . The type likelihood $p\left(\boldsymbol{z}_{k} \mid c_{i}, \boldsymbol{z}_{1: k-1}\right)$, which is a function of $c_{i}$ for a fixed $\boldsymbol{z}_{k}$, can be further expanded over selected features $f_{k}$, e.g. emitters given off by a noncooperative aircraft, as

$$
p\left(\boldsymbol{z}_{k} \mid c_{i}, \boldsymbol{z}_{1: k-1}\right)=\sum_{f_{k}} p\left(\boldsymbol{z}_{k} \mid f_{k}, \boldsymbol{z}_{1: k-1}\right) p\left(f_{k} \mid c_{i}\right)
$$

where $\boldsymbol{z}_{k}$ and $c_{i}$ are assumed to be conditionally independent given features $f_{k}$ and $\boldsymbol{z}_{1: k-1}$. We also assume $f_{k}$ and $\boldsymbol{z}_{1: k-1}$ are conditionally independent given $c_{i}$. Here the feature likelihood $p\left(\boldsymbol{z}_{k} \mid f_{k}, \boldsymbol{z}_{1: k-1}\right)$ represents the uncertain mapping that extracts feature $f_{k}$ from observed data $\boldsymbol{z}_{k}$; while $p\left(f_{k} \mid c_{i}\right)$ defines the uncertain mapping II from the type space to the feature space.

From a purely probabilistic viewpoint, the type likelihood $p\left(\boldsymbol{z}_{k} \mid c_{i}, \boldsymbol{z}_{1: k-1}\right)$, feature likelihood $p\left(\boldsymbol{z}_{k} \mid f_{k}, \boldsymbol{z}_{1: k-1}\right)$, and feature mapping $p\left(f_{k} \mid c_{i}\right)$ are all conditional probabilities. In statistics, a likelihood function (often simply the likelihood) is a function of the parameters of a statistical model, defined as follows: the likelihood of a set of parameter values given some observed outcomes is equal to the probability of those observed outcomes given those parameter values $[10,11]$. It is natural that the uncertain mapping be modeled as a statistic function since sensor observation is usually a measure of feature with corruption noise. However it is usually very difficult to assign an exact probability to the uncertain mapping II. Let us take target recognition using ESM data for example. Assume a noncooperative target being recognized belongs to type set $C=\left\{c_{1}, c_{2}\right\}$. It will at a certain time instant give off one kind of emitter $f$. The possible emitter set of type 1 target is $\left\{f^{1}, f^{3}, f^{4}, \phi\right\}$, where $\phi$ means emitters are off; the emitter set of the type 2 target is $\left\{f^{1}, f^{2}, \phi\right\}$. Though it is often possible to get the emitter set for either type of target, it is hard to tell the usage probability of each emitter category for every target type. This is the motivation for us to seek a possibility representation of the uncertain mapping II .

We draw the ideal from [6], which interprets the membership function of a fuzzy set as a conditional probability. Given $p\left(f_{k} \mid C\right)=1$ as an extension, $p\left(f_{k} \mid c_{i}\right)$ defines a possibility distribution on $C=\left\{c_{i}\right\}$. It is stressed in [6] that $p\left(f_{k} \mid c_{i}\right)$ represents the conditional probability of the conditional event $f_{k} \mid c_{i}$ as "a whole" and that this is not necessarily additive with respect to the conditioning events $\left\{c_{i}\right\}$. If $p\left(f_{k} \mid c_{i}\right)$ is denoted by $\pi_{f_{k}}\left(c_{i}\right)$, Eq. (2) can then be rewritten as

$$
\pi_{z_{k} \mid z_{1: k-1}}\left(c_{i}\right)=\alpha_{1} \sum_{f_{k}} p\left(z_{k} \mid f_{k}, z_{1: k-1}\right) \pi_{f_{k}}\left(c_{i}\right)
$$

where $\pi_{z_{k} \mid z_{1: k-1}}\left(c_{i}\right)=\alpha_{1} p\left(z_{k} \mid c_{i}, z_{1: k-1}\right)$ is the possibility that the attribute observation $\boldsymbol{z}_{k}$ is from the target $c_{i}$ given the observation sequence $\boldsymbol{z}_{1: k-1}$, and the mapping $\pi_{f_{k}}\left(c_{i}\right)$ is the possibility that the feature $f_{k}$ is from the target $c_{i}$. Scale factor $\alpha_{1}$ is applied in Eq. (3) so that the maximum value of $\pi_{z_{k} \mid z_{i k-1}}\left(c_{i}\right)$ over $C$ is assured to be 1 , which is one of the requirements of a possibility distribution. Because Eq. (1) holds for any value proportional to $p\left(\boldsymbol{z}_{k} \mid c_{i}, \boldsymbol{z}_{1: k-1}\right)$, considering the effect of the normalization constant $\alpha$, Eq. (1) can then be rewritten as

$$
p\left(c_{i} \mid \boldsymbol{z}_{1: k}\right)=\alpha p\left(c_{i} \mid \boldsymbol{z}_{1: k-1}\right) \pi_{z_{k} \mid z_{1: k-1}}\left(c_{i}\right)
$$

Eq. (4) provides a natural fusion of probability and possibility by reinterpreting Bayes' rule (1). Eqs. (3) and (4) are named possibilized Bayesian classifier. One of its merits is that the possibility assignment of $\pi_{f_{k}}\left(c_{i}\right)$ is very flexible compared with its probability assignment $p\left(f_{k} \mid c_{i}\right)$, because the feature database defining $\pi_{f_{k}}\left(c_{i}\right)$ has an open structure. For example, when new target type or emitter category is to be included for target recognition, we only need to add some new feature rules to the database of the proposed classifier. But for the conventional Bayesian classifier, we need to reconstruct the whole feature database and maintains its conformity so that the sum of $p\left(f^{j} \mid c_{i}\right)$ over $f^{j}$ is 1 , which is inconvenient.

\section{Simulation EXAMPLE}

Our simulation continues the same toy example as introduced in Section 2. ESM observation sequence generation is based on the emitter usage probability and confusion matrix. The emitter usage probability, $p\left(f^{j} \mid c_{i}\right)$, i.e., the probability of emitter $j$ given off by a type $i$ target, is given in Table 1 . The confusion process, an instantiation of $p\left(\boldsymbol{z}_{k} \mid f_{k}, \boldsymbol{z}_{1: k-1}\right)$, is defined as

$$
p\left(\text { declare } f^{j} \mid \text { detect } f^{i}\right)=\left\{\begin{array}{ll}
0.7 & i=j \\
0.1 & i \neq j
\end{array} i, j \in\{1,2,3,4\}\right.
$$

Note that if the emitter on the target is off, then no emitter will be declared. The noncooperative target is recognized using both the Bayesian classifier and the proposed classifier. The initial probabilities of the target type, $p\left(c_{i} \mid \boldsymbol{z}_{0}\right)$, are set to be equal. The emitter usage probability used by the Bayesian classifier is from Table 1 (Classifier 1) or Table 2 (Classifier 2). For Classifier $1, p\left(f^{j} \mid c_{i}\right)$ is exactly the same as that for data generation. For Classifier 2, it represents that a certain target type has an equal probability of producing every emitter category since the exact probability assignment is not know. For the proposed Classifier $3, p\left(f^{j} \mid c_{i}\right)=\pi_{f^{j}}\left(c_{i}\right)$ is defined as in Table 3, which indicates for example that $f^{1}$ can be from target $c_{1}$ or target $c_{2}$ with identical possibility of 1 . 
TABLE I. $\quad p\left(f^{j} \mid c_{i}\right)$ FOR CLASSIFIER 1

\begin{tabular}{|l|c|c|c|c|c|}
\hline & $\boldsymbol{f}^{\mathbf{1}}$ & $\boldsymbol{f}^{\mathbf{2}}$ & $\boldsymbol{f}^{\mathbf{3}}$ & $\boldsymbol{f}^{\mathbf{4}}$ & $\boldsymbol{\sigma}$ \\
\hline$c_{1}$ & 0.54 & 0.00 & 0.24 & 0.12 & 0.10 \\
$c_{2}$ & 0.45 & 0.45 & 0.00 & 0.00 & 0.10 \\
\hline
\end{tabular}

TABLE II. $\quad p\left(f^{j} \mid c_{i}\right)$ FOR CLASSIFIER 2

\begin{tabular}{|l|c|c|c|c|c|}
\hline & $\boldsymbol{f}^{\mathbf{1}}$ & $\boldsymbol{f}^{\mathbf{2}}$ & $\boldsymbol{f}^{\mathbf{3}}$ & $\boldsymbol{f}^{\mathbf{4}}$ & $\boldsymbol{\sigma}$ \\
\hline$c_{\mathbf{1}}$ & 0.25 & 0.00 & 0.25 & 0.25 & 0.25 \\
$\boldsymbol{c}_{\mathbf{2}}$ & 0.33 & 0.33 & 0.00 & 0.00 & 0.33 \\
\hline
\end{tabular}

TABLE III. $\quad \pi_{f^{j}}\left(c_{i}\right)$ FOR CLASSIFIER 3

\begin{tabular}{c|c|c|c|c|c}
\hline & $\boldsymbol{f}^{\mathbf{1}}$ & $\boldsymbol{f}^{\mathbf{2}}$ & $\boldsymbol{f}^{\mathbf{3}}$ & $\boldsymbol{f}^{4}$ & $\boldsymbol{0}$ \\
\hline$c_{1}$ & 1 & 0 & 1 & 1 & 1 \\
$c_{2}$ & 1 & 1 & 0 & 0 & 1 \\
\hline
\end{tabular}

Figs. 2 and 3 are average probabilities of 100 runs for recognizing target $c_{1}$ and $c_{2}$, respectively. Fig. 2 shows that Classifier 2 is a little pessimistic in recognizing the target $c_{1}$ while the proposed Classifier 3 is a little optimistic, but both have competitive performance as Classifier 1 which uses the exact emitter usage probability as that for data generation. Contrary conclusion can be drawn from Fig. 3. The overall performances of the Bayesian classifier and the proposed classifier are close.

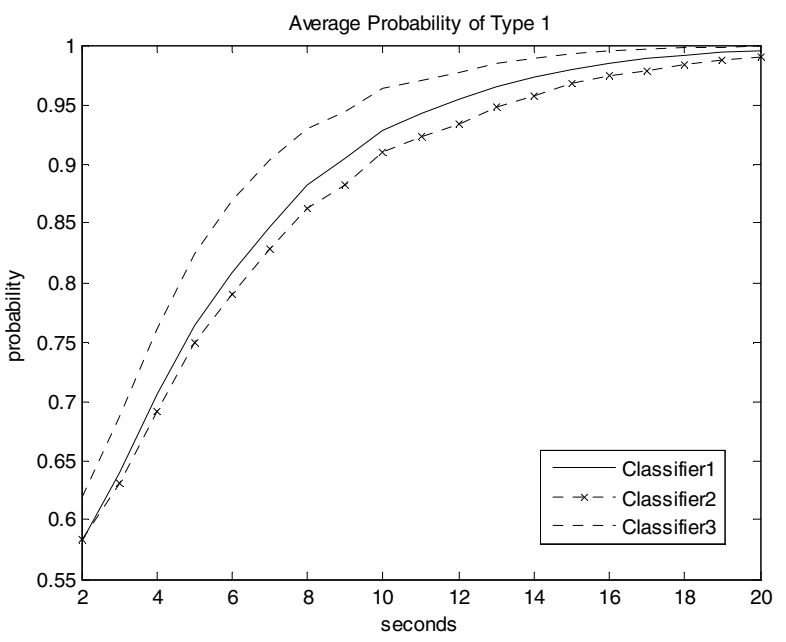

Figure 2. Recognizing $c_{1}$ target.

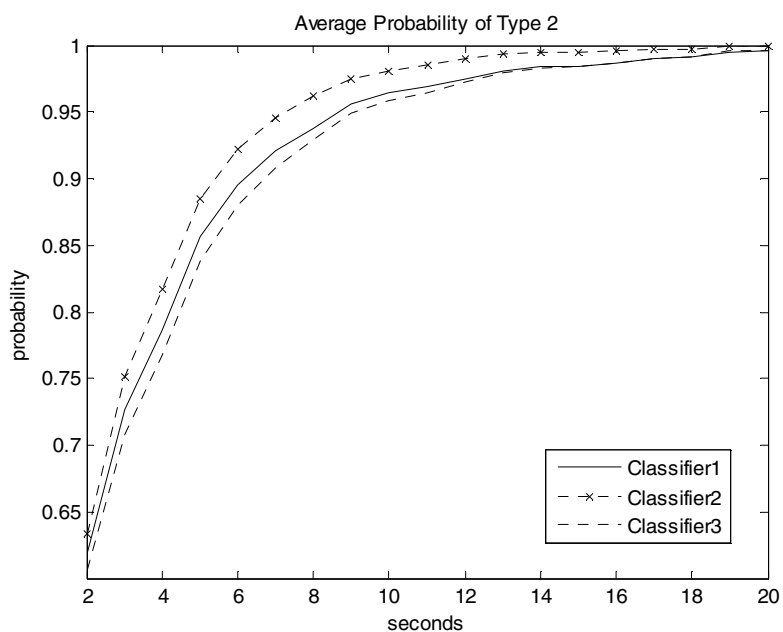

Figure 3. Recognizing $c_{2}$ target.

\section{CONCLUSIONS}

It is more natural and convenient to model the uncertainty involved in target recognition using the so called possibilized Bayes' Theorem, which has competitive recognition performance with the conventional Bayesian classifier and the merit of an open structure of feature database. Future work is to test the proposed method using more application oriented data.

\section{REFERENCES}

[1] A. K. Jain, R. P. W. Duin, and J. Mao, Statistical pattern recognition: a review, IEEE Trans. On Pattern Analysis and Machine Intelligence, 22 (2000) 4-37.

[2] H. Leung, J. Wu, Bayesian and Dempster-Shafer target identification for radar surveillance, IEEE Trans. on Aerospace and Electronic Systems, 36(2000): 432-447.

[3] F. Delmotte, P. Smets, Target identification based on the transferable belief model interpretation of Dempster-Shafer model, IEEE Trans. On Systems, Man, and Cybernetics - Part A: Systems and Humans, 34 (2004) 457-471.

[4] G. Bailadora, G. Triviño, Pattern recognition using temporal fuzzy automata, Fuzzy Sets and Systems, 161 (2010) 37-55.

[5] S.S. Young, P.D. Scott, N.M. Nasrabadi, Object recognition using multilayer Hopfield neural network, IEEE Trans. On Image processing, Mar. 1997, 357-372.

[6] G. Coletti, R. Scozzafava, Conditional probability, fuzzy sets, and possibility: a unifying view, Fuzzy Sets and Systems, 144 (2004) 227249.

[7] D. Dubois, S. Moral and H. Prade, A semantics for possibility theory based on likelihoods, Journal of Mathematic Analysis and Applications, 205 (1997) 359-380.

[8] S. F. Thomas, Possibilistic uncertainty and statistical inference, ORSA/TIMS Meeting, Houston, Texas, 1981

[9] W. Mei, G.-L. Shan, Y.-F. Wang, A Second-order uncertainty model for target recognition using kinematic Data, Information Fusion, 12 (2011) $105-110$.

[10] http://en.wikipedia.org/wiki/Likelihood_function, Likelihood function

[11] http://en.wikipedia.org/wiki/Bayes\%27_theorem, Bayes' theorem 